\title{
Agent-Based Real-Time Assembly Line Management for Wireless Job Shop Environment
}

\author{
Yingfeng Zhang, George Huang, Ting Qu and Jun Hong
}

\begin{abstract}
Recent developments in wireless technologies have created opportunities for developing next-generation manufacturing systems with real-time traceability, visibility and interoperability in shop floor planning, execution and control. This paper discusses how to deploy wireless and intelligent technologies to convert physical objects in manufacturing systems into smart objects to introduce and improve the interoperability and visibility between them and thus with manufacturing decision support systems. A reference architecture for wireless manufacturing (WM) is proposed where three types of smart objects are identified. At the same time, the concept of smart object agent (SOA) is presented and the corresponding framework of smart objects management system (SOMS) is constructed. Under this framework and the concept of SOA, a SOA-based WM environment is studied and demonstrated using a near real-life simplified product assembly line for the collection and synchronization of the real-time field data from manufacturing workshops.
\end{abstract}

\section{INTRODUCTION}

W ITH the increasing competitiveness and globalization of today's business environment, the supply chain of a company has become more complex and the manufacturing processes have become more advance, but products have to be manufactured in higher varieties and smaller batches. It is essential to adapt advanced manufacturing technologies and approaches (both software and hardware) to cope with the highly dynamic manufacturing requirements. In the recent decades, rapid developments in wireless sensors, communication and information network technologies (e.g. radio frequency identification - RFID or Auto-ID, Bluetooth, Wi-Fi, GSM, and infrared) have nurtured the emergence of Wireless Manufacturing (WM) as core Advanced Manufacturing Technology (AMT) in next-generation manufacturing systems (NGMS).

Real-time visibility and interoperability have been considered core characteristics of next-generation manufacturing systems [1]. Pilot projects have recently been

Manuscript received May 24, 2010. (Write the date on which you submitted your paper for review.) This work was supported in part by the HKSAR ITF (GHP/042/07LP) grant, National Science Foundation (50805116) and 863 Hi-Tech Research Program (2009AA04Z147) of China.

Dr. Yingfeng Zhang is with the University of Hong Kong and Xi'an Jiaotong University, China (Corresponding author: phone: 00-852-28592592; e-mail: xjtuzyf@, hku.hk).

Dr. George Huang is with the University of Hong Kong, Hong Kong, China (e-mail: gqhuang@hku.hk).

Dr. Ting Qu is with the University of Hong Kong, Hong Kong, China (e-mail: quting@hku.hk).

Dr. Jun Hong is with the Xi'an Jiaotong University, Xi'an, Shaanxi, 710049, China (e-mail: jhong@,mail.xjtu.edu.cn). implemented and reported (see various whitepapers and reports at http://www.autoidlabs.com/ researcharchive/ for more descriptions). The progress of Wireless Technologies such as RFID and AutoID applications in the "manufacturing scenario" has been noticeable although limited. As early as in early 1990s, Udoka [2]-[3] has discussed the roles of Auto ID as a real-time data capture tool in a computer integrated manufacturing (CIM) environment. Early RFID manufacturing applications have been briefly quoted in Brewer, Sloan, and Landers [4]. Johnson [5] presents a RFID application in a car production line. The website http:/www.productivitybyrfid.com/ also provides a few links to real-life pilot cases. More recently, the Cambridge Auto ID Lab has launched an RFID in Manufacturing Special Interest Group (SIG) (http://www.aero-id.org/). Zhang et al. [6] have designed and developed a RFID-based smart Kanban system for shop-floor WIP management. Based on RFID technologies, Huang et al. [7] build up a real-time manufacturing information system for controlling flows of information and materials of the entire shop-floor. Zhang et al. (2008) develops a RFID-based smart Kanban system for work-in-progress (WIP) management. An Agent-based workflow management for RFID-enabled real-time reconfigurable manufacturing has been described by Zhang et al. [8].

The concept of agent has been widely accepted and developed in manufacturing applications because of its flexibility, re-configurability, and scalability [9]-[11]. An agent based concurrent design environment [12] has been proposed to integrate design, manufacturing and shop-floor control activities. A compromising and dynamic model in an agent-based environment [9] has been designed for all agents carrying out their own tasks, sharing information, and solving problems when conflicts occur. Some mobile agent-based systems [13]-[14] have been applied to the real-time monitoring and information exchange for manufacturing control. Jia et al [15] proposed an architecture where many facilitator agents coordinate the activities of manufacturing resources in a parallel manner. Jiao et al. [16] applied the MAS paradigm for collaborative negotiation in a global manufacturing supply chain network. Besides, in various kinds of applications such as online task coordination and monitoring [17], and supply chain negotiation [18], the agent-based approach has played an important role to achieve outstanding performance with agility [19].

However, it becomes particularly challenging to integrate 
the advantage of wireless technologies and agent based methods. On one hand, wirelessly networked sensors facilitate the automatic collection and processing of real-time field data in the manufacturing processes, and reduce and avoid the error-prone, tedious manual activities. On the other hand, agent-based system enables relevant activities more flexible, intelligent and collaborative especially in distributed networked environment. Therefore, a concept of smart object agent (SOA), using agent theories to wrap smart object (wireless sensors can be seen as a kind of smart object), is proposed and a web service framework is applied to achieve the ideas presented in this paper.

\section{OVERVIEW OF REAL-TIME WIRELESS MANUFACTURING}

This section presents a conceptual framework for wireless manufacturing. It serves several purposes. Firstly, it helps defining vertical and lateral manufacturing visibility and interoperability. Secondly, it highlights potential application areas where WM solutions have roles to play. Thirdly, it defines general functionality of typical WM solutions in appropriate decision areas. Finally, it provides us with a foundation to propose a technology roadmap for WM framework.

In this WM framework, decision processes of a manufacturing enterprise can be conveniently divided into several levels and each can be further decomposed. This has been widely used in the field of Computer Integrated Manufacturing system and adopted for establishing the ISA -95 standard. The high-level planning layer is related to the enterprise within the supply chain and typical application systems include advanced planning and scheduling (APS) modules provided in ERP system. The low-level control layer includes control and automation systems that manage equipment and devices such as robots, forklifts, etc. The automation-level is more concerned with interoperations between equipment. This control level can be further decomposed into the automation and equipment levels. The equipment-level control is mainly addressed by equipment controllers. The middle-level execution layer links the planning and control layers to actually fulfil manufacturing orders at the shop-floor level and is typically represented by manufacturing execution systems (MES). Cutting through the three layers is manufacturing performance analytics and diagnostics system.

Visibility and interoperability is bi-directional, not only top-down in the manufacturing decision hierarchy from the higher to lower layers but also bottom-up feedback to close the loop. Both vertical and lateral visibility and interoperability are enabled using several innovative concepts and constructs. They include:

- Agent-based federation. All third-party computational resources and intelligences are federated through agents that interact with each other through standardized mechanisms and messages. All agents are implemented as web services and UDDI facilities are adopted and extended to form agent management systems. Three types of agents are involved in this reference framework: enterprise application agents (EAA), smart object agents (SOA) and work-in-progress agents (WIPA). The first two types of agents are lightweight to provide federal services while the third type of agents is heavyweight.

- Smart objects (SO) are innovative constructs as a result of equipping manufacturing resources (personnel, products, plant and equipment, and processes) with wireless devices and intelligence logics so that they can sense, identify, communicate, decide and act. Smart objects are federated by smart object agents (SOA), hosted at a computational platform called Smart Object Platform (SOP), and managed by a smart object management system (SOMS).

- Third-party enterprise application systems (EAS), whether at the planning and scheduling level or at the execution level or of analytics type, can be considered as special type of smart software objects with much more sophisticated data and decision models. Therefore, they can be federated by agents in the similar way as smart objects, resulting in corresponding constructs as EAA (enterprise application agent), EAN (Enterprise Application Network also known as enterprise knowledge and decision network).

- Work-In-Progress Agent (WIPA) forms the WIPN (WIP network). WIPA and WIPN are fully visible and interoperable because they directly interact with smart object agents (SOA) and EAA (enterprise application agents) if implemented. WIPA are heavyweight agents because they are themselves enterprise application systems with full operational logics and decision models.

- wipML (Work-In-Progress Markup Language) contains and extends the set of standards in a pragmatic and innovative manner for the definition and operation (e.g. messages) of agents at all levels.

\section{AGENT BASED SOMS}

This section discusses the web-service based architecture and corresponding working logic of SOMS as seen in Fig.1. The proposed SOMS includes a number of main web-service nodes which are described as follows.

\section{A. SOMC (Smart Object Management Centre) Service Node}

For each enterprise, there must exist one and only SOMC, through which SOP is defined and created. Generally, SOMC is responsible for managing the operation of the corresponding SOP directly and that of smart objects indirectly. It consists of four key components:

- Smart Object Registry (SOR) records the basic information of three types (master, slave and sensor) of 
smart objects and provides its information for other components or service nodes.

- Smart Object Workflow Management (SOWM) is used to define and manage the workflow files of the smart object's work process, and enterprise can define and edit its workflow files according to their specific business process.

- Smart Object Rule Management (SORM) is used to define and manage the rules of the smart object's event, and enterprise can also define and edit its rules according to their specific requirement.

- Monitor \& Control: Once the SOA works, supervisor or manager can monitor and control it through SOMC. Monitor component shows the real-time working status. Control component provides the supervisor with the privilege of operating SOA directly when exception occurs.

\section{B. agentUDDI Service Node}

agentUDDI Service Node performs functions similar to those of standard UDDI (Universal Description, Discovery and Integration), that is a platform-independent framework for describing services, discovering businesses, and integrating business services through Internet. It consists of five components:

- Publish \& Search: This component is used to issue SOAs as web services which can be easily found and communicated by using WSDL and SOAP.

- Business Information: This component is contained in a businessEntity structure which contains information about the business that has published the service, such as business name, description, identifiers etc.

- Service Information: This component describes a group of web services which are contained in a businessService structure and groups a set of Web services related to either a business process or group of services.

- BindingTemplate: This component provides information required to invoke a web service, such as network access point, supported interfaces, etc.

- tModel: This component describes the specifications for services. The bindingTemplate indicates the specifications or interfaces. Such a reference is called a tModelKey, and the data structure encapsulating the specification information is called a tModel.

\section{SOP Service Node}

Generally, there are many smart objects in an enterprise. In order to easily manage and control, smart objects have similar function or at the same location are grouped and each group is physically hosted on a computer. The computer is the edge host or server of the smart objects. Smart objects and their host together form an SOP (Smart Object Platform) which includes four components:

- Monitor: During the execution period, the real-time information (e.g. working status, the current event and binding information between SOA and WIPA or other agents) of SOA can be monitored through SOP.

- Maintenance: Provides some remote methods for maintenance operators to correct software exceptions through internet.

- Synchronization: This component provides some synchronized methods for SOP to get the real-time data from SOA.

- Interface: Similar to the interface of SOMS, this component acts as a communication bridge among SOA, SOP, SOMS and agentUDDI.

\section{SOA Service Node}

As discussed above, for each smart master (not slave or sensor) object (SMO), there is a corresponding software agent as an ambassador representing this object in the SOP. Smart slave (SVO) and sensor (SSO) objects do not have representative agents because they do not communicate directly with other enterprise application systems (EAS). Agents are intelligent enough to manage their own internal affairs including interactions internally with their corresponding smart objects and externally with enterprise application systems (EAS) (normally through their agents). It consists of five components (Binding, Workflow, Rules, Driver and Lifecycle) to enable SOA has following capabilities:

- Acts as the middleware for its corresponding SO. The agent maintains the software driver and basic configuration information of its smart object.

- Provides a mechanism for incorporating and maintaining business logics specific to the smart object. Typical business logics for individual smart objects are their workflows.

- Contains capability, capacity and loading profiles of the smart object. Such information is useful for real-time scheduling and other decision activities.

- Contains rules for configuring the behaviors of the smart object and its data format to be exchanged with other devices and systems.

- Is able to perform a series of functions such as filtering, aggregation, and counting of tag data.

- Knows who and how to react upon an internal SO event and/or an external event.

\section{E. WIPA Service Node}

WIP gateway agents (WIPA) are the key concept extensively used to construct the WIP gateway facilities. WIPA are real-time decision support systems. Generally speaking, for each major decision process, there is a WIPA. The WIPA community expands when more decision processes are considered. If a manufacturer has not yet implemented ERP and MES, built-in gateway facilities can be used as simplified EAS. In this sense, it is not a prerequisite for a manufacturer to invest in any EAS. A WIPA interact with one or all three groups of clients: (1) the human users, (2) SOA, and (3) EAA. WIPA are implemented as web services 
including four key components:

- View Model: This component allows the user to issue instructions and then converts them into a wipML message. The wipML message is sent to the corresponding WIPA which will reason and take appropriate actions and finally returns a wipML response message.

- Real-time Application Service: This component is real-time EASs with built-in visibility and interoperability. It is able to interact with SOA and EAA and convert their segment data into the meaningful information. Simple editing functions are provided while sophisticated functions can be extended by the user enterprise itself or achieved through the integration with third-party EASs.

- Data Model: This component contains all the real-time shop-floor information structured in a standard format. The dynamic data is obtained and collated from SOAs according to the chosen decision logic which is already reflected by the standard data model.

- Bind Model: This component provides WIPA with the capability of binding the services of the chosen SOAs. It acts as an interface that connects the output of SOAs (Smart Object Agent) to the input of WIPA.

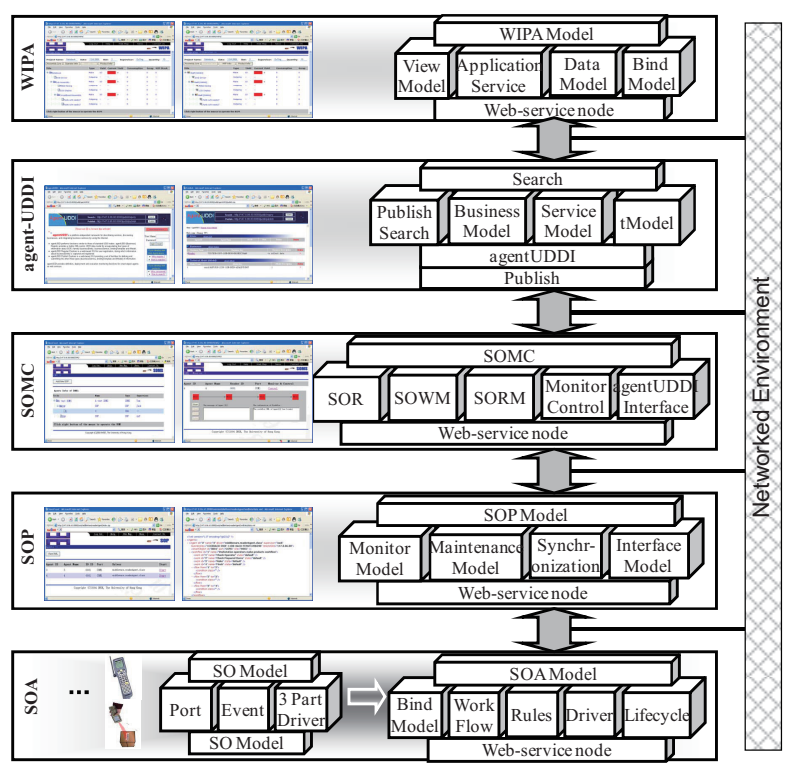

Fig. 1. Web-service based architecture of SOMS.

\section{Illustrative ASSEMBLy LINE AND SMART OBJeCtS}

The conceptual WM framework was only proposed and summarized briefly in the preceding section. Its details are yet to be presented in a separate work. This section exemplifies and instantiates the proposed WM framework with a simple application in an assembly line. This proof-of-the-concept test-bed serves the purpose of demonstrating how the proposed WM framework would work in an industrial environment, gaining insights about requirements of WM solutions, and highlighting further issues for research and development of WM solutions. The study is based on a simplified motivating scenario shown in Fig.2.

In order to create a smart objects-based manufacturing shop-floor, firstly, it is necessary to identify shop-floor objects to which RFID tags and/or readers and/or other types of wireless devices are attached. In this case, two types of smart objects, lead objects and smart sensor objects, are utilized, which are deployed as follows:

- RFID readers (Lead Objects) installed on workstation: It is assumed that each workstation in the assembly line is equipped with one RFID reader which is able to read tags attached to different objects.

- RFID readers (Lead Objects) installed on storeroom: RFID readers are available at the workshop storeroom for the tools and materials, and in management points (e.g. workshop entrances) and offices.

- RFID readers (Lead Objects) installed on trolleys: Trolleys with which pallets are moved across the shop-floor are equipped with a reader and a PC.

- Staff card (Sensor Objects): Staff members have their staff cards which can be read by the RFID readers.

- Tools card (Sensor Objects): Critical tools and equipments are also attached with RFID tags. They are considered critical and therefore deserve tags due to their important roles.

- Critical component card (Sensor Objects): One of the components in the product assembly is considered critical and each critical component is attached with an RFID tag. This tag, as a mobile memory of the "smart" WIP products, plays important roles throughout the assembly process and even retained for subsequent supply chain applications.

- Pallet card (Sensor Objects): RFID tags are attached to all the pallets for holding WIP materials, including components/parts, scraped materials, semi-finished subassemblies and finished product assemblies. These tags are not only used for tracking the flow of materials but also for controlling the WIP inventories. Such field information will in turn be fed back and used for production planning and scheduling.

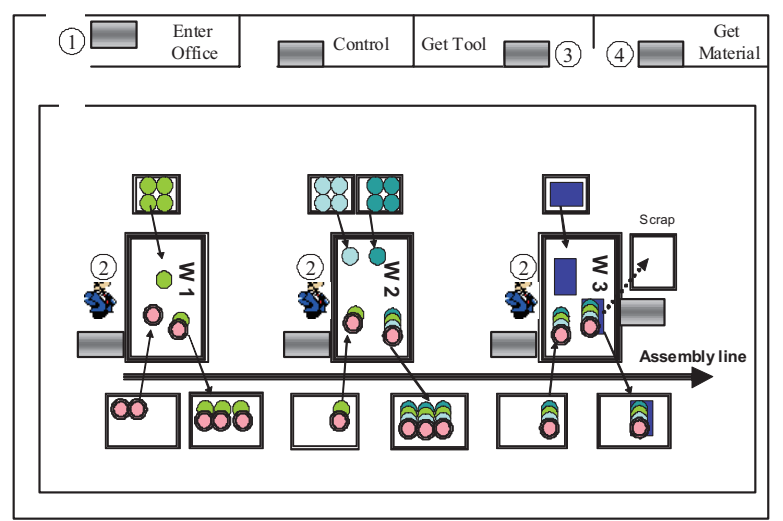

Fig. 2. Overview of the motivating assembly line. 
It is assumed that there are three SOPs (Smart Object Platform): one is used to manage the SOs of assembly line; the other is used to manage the SOs of storeroom for the tools and materials; the third one is used to manage the SO of the trolley (assume each trolley has one RFID reader). It is also supposed that each workstation or each storeroom has a display with PDA functions which can visit the internet easily.

\section{Application of SMART OBJECtS FOR REAL-TIME ASSEMBLY LINE}

\section{A. SOA Explorer}

At the workstation level, the operators are mainly responsible for both executing and controlling the operations taking place at their corresponding workstations. Fig.3. outlines an overview of assembly execution and control facilities. Once the SOAs of the workstation attached RFID readers are started from the corresponding SOP or SOMS, production operators can use SOA explorer to execute assembly plans and schedules at the level of individual workstations.

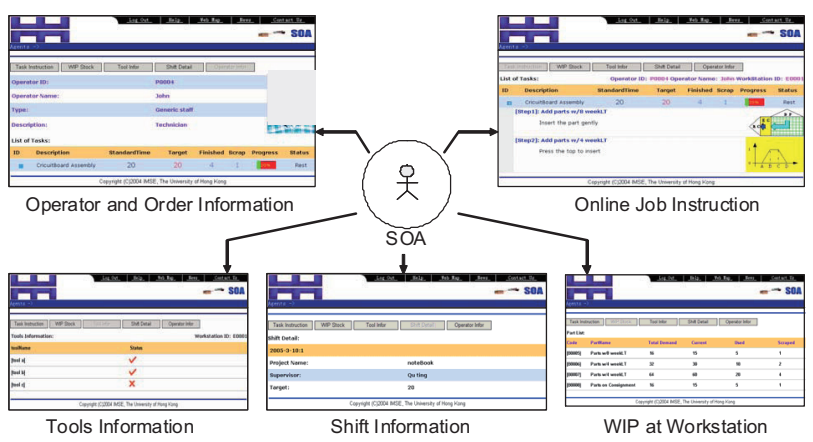

Fig. 3. Assembly execution and control at workstations.

\section{B. WIPA Explorer}

In contrast, the shop-floor manager or line supervisor is responsible for monitoring and controlling the assembly operations at the line and workstation levels. The WIPA Explorer provides facilities mainly for information display and also acts as interface with corresponding EAA (Enterprise Application Agent, e.g. ERP decision support). Therefore, its main function is to organize the real-time information captured from the SOAs of assembly line to serve different purposes. As the user interface, the WIPA explorer is where the line supervisors can sense the shop-floor changes and disturbances. The supervisors can then take corrective actions by manually using corresponding explorers. As shown in Fig.4., the WIPA explorer provides facilities for the manager/supervisor to monitor the following aspects of the assembly line:

- Master production plans and schedules

- Production status of a selected shift

- Execution status of a selected assembly schedule of a shift

- Inventories of WIP materials

- Conditions of critical tools and equipment
- Process reliability and quality records

- Staff allocation in a selected shift

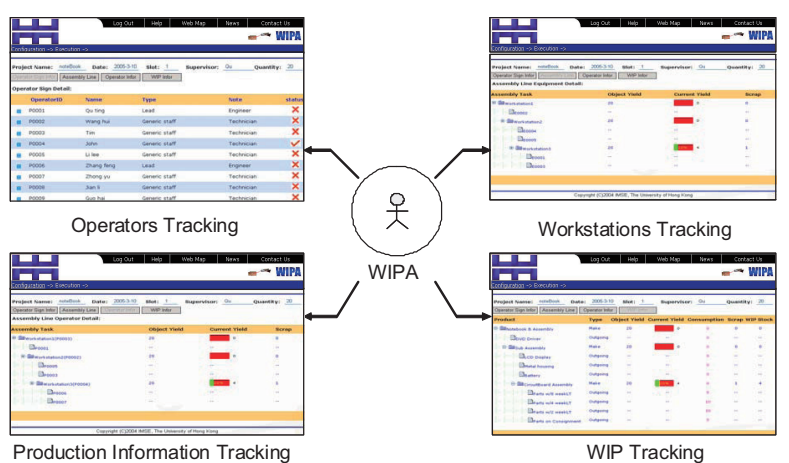

Fig. 4. Assembly monitoring and control across the line.

\section{Monitor and Control Explorer}

During the execution of SOA, some problems should be considered and solved. For example, the SOA needs to be monitored during the runtime to decrease potential errors occurred; sometimes the SOA needs to be reset remotely and be maintained online; the SOA needs to reflect its status at the workflow level, etc.

To achieve these objects, a series of facilities are developed in SOMS explorer, which provide the real-time information of each SOA for the smart objects supervisor, a staff of IT department, to monitor or control the corresponding SOA. As shown in figure 5, all the SOPs and SOAs in the enterprise are recorded and displayed as a tree mode. Through SOMS explorer, supervisor can capture the real-time workflow status of any remote SOA even operate the SOA by using the control buttons including start, resume, pause, continue and stop operations when exception occurs. Moreover, the real-time message of SOA is in favour of analysing and maintaining the corresponding smart object (e.g. RFID Reader) through internet.

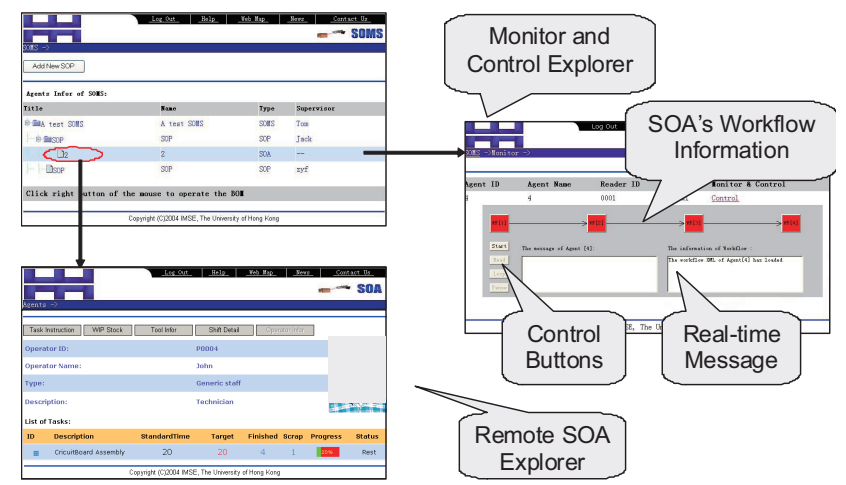

Fig. 5. SOA monitoring and control through SOMS.

\section{CONClusion}

Wireless Manufacturing (WM) is emerging as advanced manufacturing technology (AMT) which relies substantially on wireless devices such as RFID (Radio Frequency Identification) or Auto ID sensors and wireless information 
networks for the collection and synchronization of the real-time field data from manufacturing workshops. WM replaces the paper-based manual WIP tracing and tracking system by RFID-based smart devices and system, and ultimately to improve and optimize the shop-floor operational efficiency and effectiveness. The system closes the loop of production planning and control by providing real-time feedback of such sensory data for adaptive decision making.

This paper has proposed and defined the concept of smart objects and their agents in manufacturing environments. By using network and information technologies, a web service framework of SOA-based management system has been established. Under the framework, smart objects can be easily wrapped as agents that are able to not only conduct the behaviors of the corresponding SOs but also have the intelligence to interact with other SOAs, EAAs or EAS based on their workflow, rules and communication strategies. At the same time, a SOA-based assembly line is studied to illustrate how a smart assembly line is deployed, how their SOAs are defined and published and how their SOAs work and communicate with WIPA through SOMS platform. Although the study was conducted using a simplified product assembly line, findings and insights gained from the study can be generalized for other more complicated manufacturing environments. This is because more workstations can be added in order to scale up the production line, while the real-time flow of WIP materials can be managed in a more or less similar fashion. In addition, the proposed framework provides useful references for further research and development of RFID hardware devices, software middleware components, and a mechanism for enterprise data synchronization and sharing.

Further research and development are necessary if the concept of SOA and SOMS are widely implemented in enterprises. Firstly, the communication between this system and other wireless devices (e.g. Bluetooth, GSM etc.) should be studied for a WM with various wireless devices. Secondly, other important application agents (e.g. re-scheduling agent and WIP logistics agent) should be developed to enhance the real-time decision. Re-scheduling is a quite complicated problem, but it can be modelled and solved if the real-time production information could be gathered in time through this SOA-based solution. WIP logistics plays very important roles in well-complemented and enabling the production with the capability of continuity and stabilization. Another important issue is how to use workflow and rules to make the SOA enough intelligent to execute more complex actions such as self-maintenance. Finally, how to form a closed-loop manufacturing control methodology based on real-time information obtained by SOAs is also significant for production planning and shop-floor control of WM environment.

\section{ACKNOWLEDGMENT}

We are most grateful to various companies who provide technical and financial supports to this research.

\section{REFERENCES}

[1] G.Q. Huang, Y.F. Zhang, P.Y. Jiang, "RFID-based wireless manufacturing for real-time management of job shop WIP inventories", Int. J. of Advanced Manufacturing Technology, No.36, pp.752-764, 2008.

[2] S. J. Udoka, "Automated Data Capture Techniques: A Prerequisite for Effective Integrated Manufacturing System." Computers and Industrial Engineering, Vol.21, No.1-4, pp. 217-221, 1991.

[3] S. J. Udoka, "The role of automatic identification (Auto ID) in the computer integrated manufacturing (CIM) architecture," Computers and Industrial Engineering, Vol.23, No.1-4, pp. 1-5, 1992.

[4] A. Brewer, N. Sloan, and T. Landers, "Intelligent tracking in manufacturing", Int. J. of Intelligent Manufacturing, Vol. 10, No.3-4, pp. 245-250, 1999.

[5] D. Johnson, "RFID tags improve tracking, quality on Ford line in Mexico," Control Engineering, Vol.49, No.11, pp. 16-16, 2002.

[6] Y.F. Zhang, P.Y. Jiang, G.Q. Huang, "RFID-Based Smart Kanbans for Just-In-Time Manufacturing” , Int. J. of Materials and Product Technology, Vol.33, No.1-2, pp.170-184, 2008.

[7] G.Q. Huang, Y.F. Zhang, P.Y. Jiang, "RFID-Based Wireless Manufacturing for Walking-Worker Assembly Shops with Fixed-Position Layouts", Int. J. of Robotics and Computer Integrated Manufacturing, Vol.23, No.4, pp469-477, 2007.

[8] Y.F. Zhang, G.Q. Huang, T. Qu, "Agent-based workflow management for RFID-enabled real-time reconfigurable manufacturing", Int. J. of Computer Integrated Manufacturing, Vol.23, No.2, pp.101-112, 2010.

[9] R. Sikora and M.J. Shaw "A multi-agent framework for the coordination and integration of information systems", Management Science, Vol.44, No.11, pp.65-78, 1998.

[10] R. Macchiaroli and S. Riemma, "A negotiation scheme for autonomous agents in job shop scheduling", Int. J. of Computer Integrated Manufacturing, Vol.15, No.3, pp.222-232, 2002.

[11] F.P. Maturana, P. Tichy, P. Slechta, F. Discenzo, R.J. Staron and K. Hall, "Distributed multi-agent architecture for automation systems", Expert Systems with Applications, Vol.26, No.1, pp. 49-56, 2004.

[12] N. Krothapalli and A. Deshmukh, "Design of negotiation protocols for multi-agent manufacturing systems", Int. J. of Production Research, Vol.37, No.7, pp. 1601-1624, 1999.

[13] R.S. Chen, C.C. Chen, and C.C. Chang, "A Java-based mobile agent framework for enterprise applications", Int. J. of Computer Applications in Technology, Vol.15, No.6, pp.276-286, 2002.

[14] M. Shin and M. Jung, "MANPro: mobile agent-based negotiation process for distributed intelligent manufacturing", Int. J. of Production Research, Vol.42, No.2, pp.303-320, 2004.

[15] H.Z. Jia, S.K. Ong, J.Y.H. Fuh, Y.F. Zhang and A.Y.C. Nee, "An adaptive upgradable agent-based system for collaborative product design and manufacture", Int. J. Robotics and Computer-Integrated Manufacturing, Vol.20, No.2, pp.79-90, 2004.

[16] J.R. Jiao, X. You and A. Kumar, "An agent-based framework for collaborative negotiation in the global manufacturing supply chain network", Int. J. Robotics and Computer Integrated Manufacturing, Vol.22, No.3, pp.239-255, 2006.

[17] W.B. Lee and H.C.W. Lau, "Multi-agent modelling of dispersed manufacturing networks", Expert Systems with Applications, Vol.16, No.3, pp. 297-306, 1999.

[18] D.J. Wu, "Software agents for knowledge management: Coordination in multi-agent supply chains and auctions", Expert Systems with Applications, Vol.20, No.1, pp. 51-64, 2001.

[19] D. Wang, S.V. Nagalingam and G.C.I. Lin, "Development of an agent-based Virtual CIM architecture for small to medium manufacturers", Int. J. Robotics and Computer-Integrated Manufacturing, Vol.23, No.1, pp. 1-16, 2007. 